\title{
Delaying Caspase Activation by Bcl-2: A Clue to Disease Retardation in a Transgenic Mouse Model of Amyotrophic Lateral Sclerosis
}

\author{
Slobodanka Vukosavic, ${ }^{1}$ Leonidas Stefanis, ${ }^{1,2}$ Vernice Jackson-Lewis, ${ }^{1}$ Christelle Guégan, ${ }^{1}$ Norma Romero, ${ }^{1}$ \\ Caiping Chen, ${ }^{1}$ Michel Dubois-Dauphin, ${ }^{3}$ and Serge Przedborski ${ }^{1,2}$
}

\begin{abstract}
Departments of ${ }^{1}$ Neurology and ${ }^{2}$ Pathology, Columbia University, New York, New York 10032, and ${ }^{3}$ Department of Psychiatry, HUG Belle-Idee, University of Geneva School of Medicine, 1225 Geneva, Switzerland
\end{abstract}

\begin{abstract}
Molecular mechanisms of apoptosis may participate in motor neuron degeneration produced by mutant copper/zinc superoxide dismutase (mSOD1), the only proven cause of amyotrophic lateral sclerosis (ALS). Consistent with this, herein we show that the spinal cord of transgenic mSOD1 mice is the site of the sequential activation of caspase-1 and caspase-3. Activated caspase- 3 and its produced $\beta$-actin cleavage fragments are found in apoptotic neurons in the anterior horn of the spinal cord of affected transgenic mSOD1 mice; although such neurons are few, their scarcity should not undermine the potential importance of apoptosis in the overall mSOD1-related neurodegeneration.
\end{abstract}

Overexpression of the anti-apoptotic protein $\mathrm{Bcl}-2$ attenuates neurodegeneration and delays activation of the caspases and fragmentation of $\beta$-actin. These data demonstrate that caspase activation occurs in this mouse model of ALS during neurodegeneration. Our study also suggests that modulation of caspase activity may provide protective benefit in the treatment of ALS, a view that is consistent with our recent demonstration of caspase inhibition extending the survival of transgenic mSOD1 mice.

Key words: amyotrophic lateral sclerosis; apoptosis; Bcl-2; caspase; superoxide dismutase; neuronal death
Amyotrophic lateral sclerosis (ALS) is a fatal paralytic disease characterized by a progressive loss of spinal cord motor neurons (Rowland, 1995). Important insights into its pathogenesis come from the discovery that missense mutations in copper/zinc superoxide dismutase (SOD1) are linked to familial ALS (Deng et al., 1993; Rosen et al., 1993) and that overexpression of different SOD1 mutants (mSOD1) in mice replicate the clinical and pathological hallmarks of ALS (Brown, 1995). mSOD1 cytotoxicity is not triggered by a loss of enzymatic activity or by a dominant negative mechanism but rather by a gain of function (Brown, 1995) presumably related to oxidative stress (Wiedau-Pazos et al., 1996; Yim et al., 1996), protein aggregation (Durham et al., 1997), aberrant protein-protein interactions (Kunst et al., 1997), or decreased binding affinity for zinc (Estevez et al., 1999).

As illustrated below, mounting evidence indicates that mSOD1induced spinal cord motor neuron death involves, at least in part, the apoptotic molecular machinery. For instance, overexpression of mSOD1, but not of wild-type SOD1 (wtSOD1), kills cells by apoptosis in both immortalized cell lines and primary neuronal cultures (Rabizadeh et al., 1995; Mena et al., 1997). The overexpression of the anti-apoptotic protein Bcl-2 in transgenic mSOD1 mice delays the onset of ALS symptoms by retarding the loss of myelinated nerve fibers and the death of spinal cord motor neurons, thus prolonging survival (Kostic et al., 1997). Caspase-1 is activated in mSOD1-transfected neuroblastoma cells subjected to

\footnotetext{
Received July 29, 2000; revised Sept. 25, 2000; accepted Oct. 2, 2000.

This study is supported by Muscular Dystrophy Association, the Amyotrophic Lateral Sclerosis (ALS) Association, Project-ALS, the National Institute of Neurological Disorders and Stroke (Grants R01 NS38586, R29 NS37345, P50 NS38370), the United States Department of Defense (Grant DAMD 17-99-1-9471), the Lowenstein Foundation, the Smart Foundation, and the Parkinson's Disease Foundation. L.S. is a recipient of the Burroughs Wellcome Career Award in Biomedical Sciences, and C.G. is a recipient of a scholarship from the Institut National de la Santé et de la Recherche Médicale (France). We are grateful to Drs. A. Srinivasan (Idun Pharmaceutical, La Jolla, CA) for providing the CM1 antibody, G. M. Cole (UCLA, Los Angeles, CA) for providing the fractin antibody, and P. Pasinelli for insightful comments on this manuscript.

Correspondence should be addressed to Dr. Serge Przedborski, Departments of Neurology and Pathology, BB-307, Columbia University, 650 West 168th Street, New York, NY 10032. E-mail: SP30@Columbia.edu.

Copyright (C) 2000 Society for Neuroscience $0270-6474 / 00 / 209119-07 \$ 15.00 / 0$
}

oxidative stress and in spinal cords of affected transgenic mSOD1 mice (Pasinelli et al., 1998), whereas overexpression of a dominant negative mutant of caspase-1 prolongs survival of transgenic mSOD1 mice (Friedlander et al., 1997), and, as we showed recently, chronic infusion of a pan-caspase inhibitor to these mice provides significant neuroprotection (Li et al., 2000).

By virtue of its downstream position within the caspase cascade, caspase-3 activation plays a critical role in the apoptotic process (Hakem et al., 1998; Kuida et al., 1998). In support of its important role in apoptosis in many types of neurons is the demonstration that mutant mice deficient in caspase-3 exhibit severe brain abnormalities because of a marked decrease in developmental neuronal death (Kuida et al., 1996). Activation of caspase-3 also occurs in several experimental models of acute neurological disorders such as strokes, head trauma, and epilepsy; caspase-3 inhibition, by specific synthetic antagonists, is beneficial in all of these conditions (Gillardon et al., 1997; Yakovlev et al., 1997; Namura et al., 1998). However, whether caspase-3 is activated in a chronic neurodegenerative process such as in ALS is not yet known.

In the present study, we demonstrate (1) that caspase- 1 and caspase- 3 are activated sequentially in the spinal cords of affected transgenic mSOD1 mice, (2) that activated caspase-3 is localized within neurons of the anterior horn that exhibit apoptotic features, and (3) that overexpression of Bcl-2 delays caspase activation in these animals. Collectively, our data indicate that activation of caspase- 3 is a prominent feature of mSOD1-induced neurodegeneration.

\section{MATERIALS AND METHODS}

Animals. Three lines of hemizygote transgenic mice were used: (1) line B6SJL-TgN (SOD1-G93A)1Gur (Jackson Laboratories, Bar Harbor, ME), which carries the point mutation Gly $\rightarrow$ Ala at codon 93 of the human SOD1 gene and expresses $\sim 18$ copies of human mSOD1 gene (Gurney et al., 1994); (2) line B6SJL-TgN (SOD1-G93A)2Gur (Jackson Laboratories), which carries $>10$ copies of human wild-type SOD1 gene (Gurney et al., 1994); and (3) line bcl-2-57, which carries $>16$ copies of the human $b c l-2$ gene (Martinou et al., 1994) and which were back-crossed more than five times with B6SJL mice (Jackson Laboratories). Also, some transgenic mSOD1 mice were crossed with transgenic bcl-2 to produce transgenic mSOD1/bcl-2 mice. On postnatal day 14 all mice were genotyped as performed by Kostic et al. (1997). 
Total RNA preparation and RT-PCR for caspase-1 and caspase-3 and $\beta$-actin. Total RNA from both spinal cord and cerebellum of the different groups of mice were prepared with the RNeasy kit (Qiagen, Valencia, CA) according to the manufacturer's instructions. First-strand cDNA were synthesized by using SuperScript II RNase H-reverse transcriptase (Life Technologies, Gaithersburg, MD) according to the manufacturer's instructions. Then $1 \mu \mathrm{l}$ of cDNA template was amplified by PCR in a $20 \mu \mathrm{l}$ total reaction volume containing $18 \mu \mathrm{l}$ of Supermix (Life Technologies), $10 \mathrm{fmol}$ of $\left[{ }^{32} \mathrm{P}\right] \mathrm{dCTP}$ (NEN-DuPont, Wilmington, DE), and $10 \mathrm{pmol}$ of each specific primer (Life Technologies). The caspase-3 primer sequences were 5'-GTCCAGGGAGAAGGACTCG-3' (forward) and 5'-CATCTCGCTCTGGTACGG-3' (reverse). The caspase-1 primer sequences were 5'GTGTCTTGGAGACATCTG-3' (forward) and 5'-AGCAGTGGGCATCTGTAGCC-3' (reverse). As an internal control, $\beta$-actin cDNA was coamplified with primer sequences $5^{\prime}$-CTTTGATGTCACGCACGATTTC-3' (forward) and 5'-GGGCCGCTCTAGGCACCAA-3' (reverse) All primers were mouse-specific and intron-spanning and were designed on the basis of reported sequences available from the GenBank database. To control for the specificity of the PCR reaction, we performed the reaction by using normal mouse genomic DNA and mRNA subjected to cDNA synthesis without RT; neither reaction produced caspase-1, caspase-3, or $\beta$-actin PCR products. The conditions for each PCR amplification resulted in an exponential amplification range for quantification of each mRNA. After amplification, the products were separated on a 5\% polyacrylamide gel. Bands were visualized and quantified by a Bio-Rad Phosphor Imager (Hercules, CA).

Western blot analysis of caspase-1 and caspase-3. Mouse spinal cord and cerebellum protein extracts were prepared as described (Ara et al., 1998) Proteins $(50-200 \mu \mathrm{g})$ were separated on a $10 \%$ SDS-polyacrylamide gel electrophoresis and transferred to nitrocellulose membrane as in Ara et al (1998). Blots were probed with either anti-caspase-1 p20 antibody (1:1000 M-19, Santa Cruz Biotechnology, Santa Cruz, CA) or anti-caspase-3 antibody (1:1000; PharMingen, San Diego, CA), which recognize both the pro-caspases and their cleaved products. Bound primary antibody was detected, using a horseradish-conjugated anti-IgG antibody (1:2000 dilution; Amersham, Arlington Heights, IL) and a chemiluminescent substrate (SuperSignal Ultra, Pierce Chemical, Rockford, IL). X-ray films (Kodak BioMax MS, Rochester, NY) were scanned on a HP-4C Scanjet, and bands were quantified by using the NIH-Image 1.62 software (Bethesda, MD).

Caspase-1, caspase-3, and fractin immunohistochemistry. These were performed by following our standard protocol as previously described (Kostic et al., 1997). In brief, after being fixed by perfusion, the spinal cords were dissected out from the spine on ice, post-fixed by immersion in $4 \%$ paraformaldehyde in phosphate buffer, $\mathrm{pH} 7.1$ ( $\mathrm{PB}$; for $4 \mathrm{hr}$ at $4^{\circ} \mathrm{C}$ ), cryoprotected in $20 \%(\mathrm{w} / \mathrm{v})$ sucrose in $\mathrm{PB}$, and frozen by immersion in isopentane cooled on dry ice. Frozen spinal cord samples were cut $(40 \mu \mathrm{m})$ in a cryostat; 10-20 serial sections from lumbar (L3) levels were collected in ice-cold $\mathrm{PB}$ free-floating and then successively rinsed (three times for 5 min each) in $0.1 \mathrm{M} \mathrm{PB}, \mathrm{pH} 7.4$, containing $9 \mathrm{gm} / 1 \mathrm{NaCl}$ (PBS), incubated in $3 \%$ normal serum (NS) in PBS $\left(60 \mathrm{~min}\right.$ at $\left.25^{\circ} \mathrm{C}\right)$, and incubated overnight $\left(4^{\circ} \mathrm{C}\right.$ ) in a rabbit polyclonal anti-pro-caspase-1 (Santa Cruz Biotechnology), a rabbit polyclonal anti-caspase-3 antibody (Upstate Biotechnology, Lake Placid, NY) recognizing the pro-form, the custom-made affinity-purified CM1 antibody (gift from Dr. Srinivasan, Idun Pharmaceuticals, La Jolla, CA) that selectively recognizes the $17 \mathrm{kDa}$ cleaved fragment of caspase-3 (Srinivasan et al., 1998), or the custom-made affinitypurified anti-fractin antibody (gift from Dr. Cole, Department of Medicine, UCLA, Los Angeles, CA) that selectively recognizes a $32 \mathrm{kDa} C$-termina fragment of $\beta$-actin (Yang et al., 1998). All primary antibodies were diluted in PBS containing 3\% NS. After three 5 min rinses in PBS the sections were incubated successively $\left(1 \mathrm{hr}\right.$ at $\left.25^{\circ} \mathrm{C}\right)$ in biotinylatedconjugated polyclonal anti-IgG antibody (1:200; Vector, Burlingame, CA), rinsed (three times for $5 \mathrm{~min}$ each) in PBS, incubated in horseradishconjugated avidin/biotin complex (Vector), rinsed again (three times for 5 min each) in PBS, and incubated in diaminobenzidine $/ \mathrm{H}_{2} \mathrm{O}_{2}$. Spinal cord sections from nontransgenic mouse embryos of gestational age day 20 were used as positive control for apoptotic cells. Some sections also were coincubated with anti-CM1 or fractin antibody and anti-neurofilament (1:1000, monoclonal; Sternberger Monoclonal., Baltimore, MD), GFAP (1:500, monoclonal; Boehringer Mannheim, Indianapolis, IN) or MAC-1 (1:250, monoclonal; Serotec, Raleigh, NC). Then the sections were incubated with a Texas Red-conjugated anti-mouse antibody and a biotinylated-conjugated anti-rabbit antibody with fluorescein-conjugated avidin (Vector).

Quantitative morphology. Spinal segments were identified by location of spinal roots and by the characteristic morphology of the spinal cord Because the number of CM1- and fractin-positive cells in the spinal cord at any given time was very small, we could not use our stereological method (Mandir et al., 1999); instead we have used our previously published assumption-based method (Przedborski et al., 1996), following strict guidelines (Coggeshall and Lekan, 1996) to ensure the validity of our quantification technique. All sections were counterstained with thionin. In brief, the spinal cord neuronal counts were performed manually and blinded to the mouse category (i.e., transgenic vs nontransgenic). For each mouse at least 10 CM1- and fractin-immunostained and Nissl-stained sections at L3 were analyzed by scanning the entire anterior horn on both sides at $400 \times$ magnification. For each section, right and left counts of motor neurons

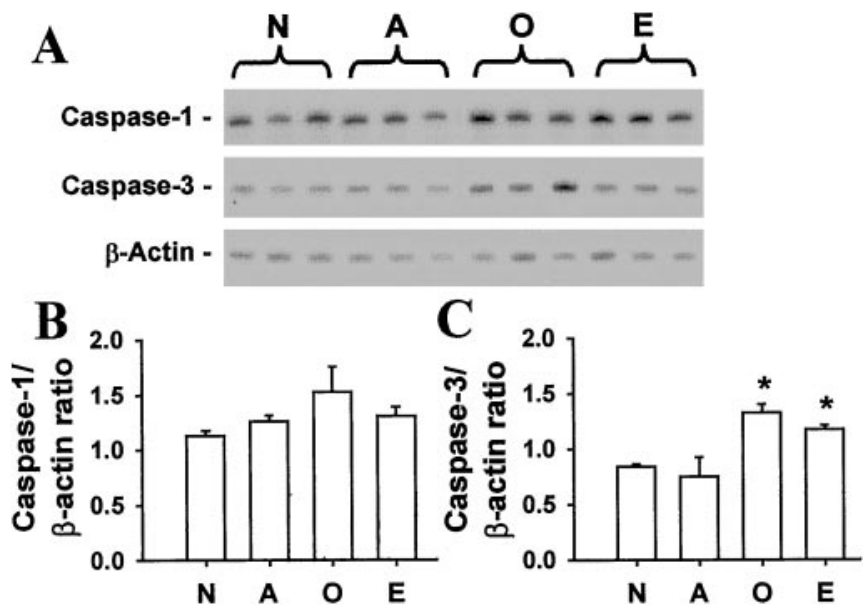

Figure 1. Caspase-1 and caspase- 3 mRNA levels are altered in the spinal cords of transgenic mSOD1 mice. $A, B$, Trend toward increased levels of caspase-1 mRNA in transgenic mSOD1 mice at the beginning of symptoms and at end-stage. $A, C$, Significant increased caspase-3 mRNA levels in transgenic mSOD1 mice at the beginning of symptoms and at end-stage; ${ }^{*} p<0.05$ higher than age-matched nontransgenic controls, Newman-Keuls post hoc test. $N$, Nontransgenic; $A$, asymptomatic; $O$, onset of symptoms; $E$, end-stage.

were averaged. The averaged number of motor neurons in each L3 section was added and then divided by the number of sections that were used to express the results as number of motor neurons per section.

Caspase- 1 and caspase- 3 activities. These were assessed in spinal cord and cerebellum of different groups of mice and at different ages by using fluorogenic assays as previously described (Stefanis et al., 1996) with minor modifications. PC -12 cell lysates, isolated from cells subjected to serum withdrawal (Stefanis et al., 1996), were used as a positive control. Proteins from tissue samples were prepared as for cell lysates, but with the inclusion in the extraction buffer of $0.1 \%$ Triton X-100. After ultracentrifugation $(160,000 \times g$ for $20 \mathrm{~min})$, proteins from the soluble fraction $(100 \mu \mathrm{g})$ were incubated at $37^{\circ} \mathrm{C}$ in $1 \mathrm{ml}$ of reaction buffer $(25 \mathrm{mM}$ HEPES, pH 7.5, 0.1\% CHAPS, $10 \mathrm{~mm}$ dithiothreitol, and $10 \%$ sucrose). Each sample was preincubated for $30 \mathrm{~min}$ at $37^{\circ} \mathrm{C}$ with or without presence of $20 \mu \mathrm{M}$ of specific inhibitor (Z-DEVD-FMK for caspase-3 and Z-YVAD-FMK for caspase-1; Enzyme Systems Products, Livermore, CA). Then the samples were incubated with fluorogenic substrates: $15 \mu \mathrm{M}$ Ac-DEVD-AFC for caspase-3 and $15 \mu \mathrm{M}$ Ac-YVAD-AFC for caspase-1 (Enzyme Systems Products). Cleavage of the substrate was monitored over time in a SLM 8000 fluorimeter (excitation $400 \mathrm{~nm}$, emission $505 \mathrm{~nm}$ ) as previously described (Stefanis et al., 1996).

Statistical analysis. All values are expressed as the mean \pm SEM. Differences among means were analyzed with one- or two-way ANOVA with time, treatment, or genotype as the independent factors. When ANOVA showed significant differences, pair-wise comparisons between means were tested by Newman-Keuls post hoc testing. In all analyses the null hypothesis was rejected at the 0.05 level.

\section{RESULTS}

\section{Time course of behavioral abnormalities in transgenic mSOD1 mice}

Consistent with our previous studies (Kostic et al., 1997), the first behavioral abnormalities in these animals, which occurred between 12 and 14 weeks of age, were a fine tremor in and posturing of at least one limb when the animal was held in the air by the tail. After the beginning of these symptoms, weakness and atrophy progressed, leading to end-stage over $\sim 8$ weeks. At that point, the animals were between 20 and 22 weeks old and were so severely paralyzed that they could not eat, drink, or move freely and thus were killed.

\section{Caspase-1 and caspase-3 mRNA levels in transgenic mSOD1 mice}

In connection to the described course of disease, we found that expression of caspase- 1 and caspase- 3 mRNA did not differ in the spinal cords of asymptomatic transgenic mSOD1 mice of 12 weeks of age as compared with age-matched nontransgenic controls (Fig. $1)$. In contrast, expression of caspase- 1 and caspase- 3 mRNA appeared elevated in both early symptomatic and end-stage trans- 


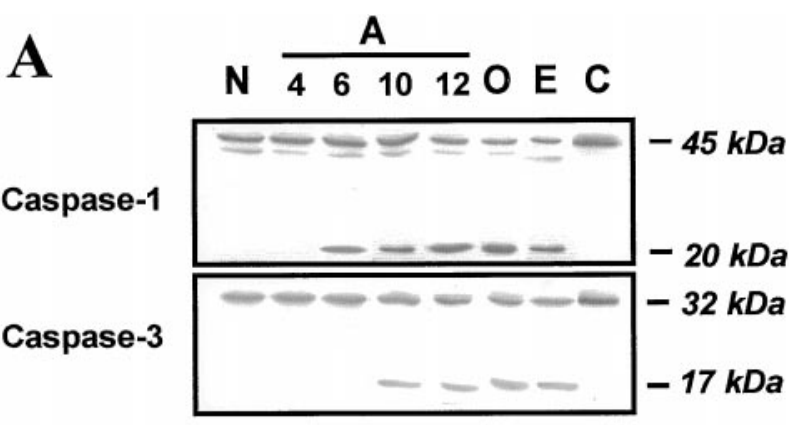

B
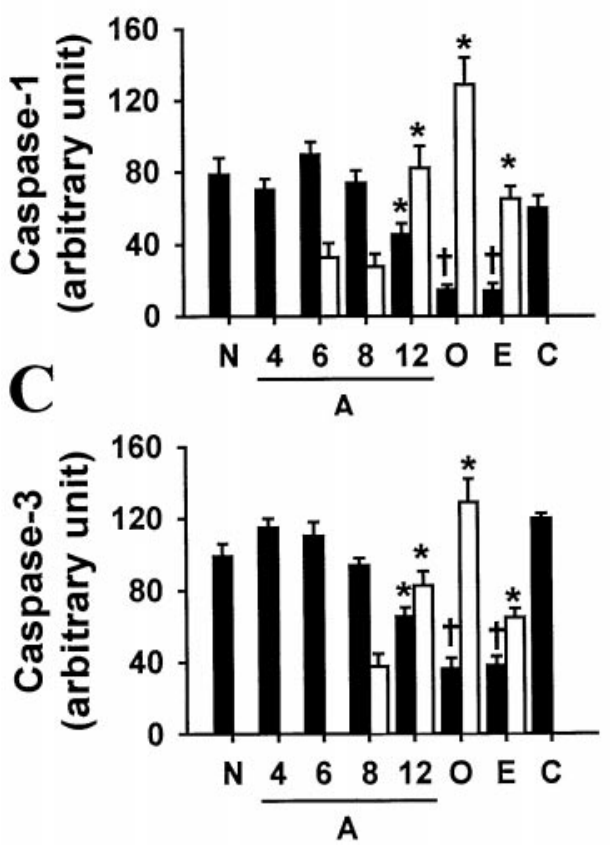

Figure 2. Activation of caspase-1 and caspase-3 in the spinal cords of transgenic mSOD1 mice. $A, B$, By 12 weeks of age the levels of procaspase-1 (45 kDa; black bars) decrease, whereas those of its cleaved fragment ( $20 \mathrm{kDa}$; white bars) increase in transgenic mSOD1 mice. $A, C, \mathrm{~A}$ few weeks later the same phenomenon occurs for pro-caspase-3 $(32 \mathrm{kDa}$; black bars) and its cleaved fragment (17 kDa; white bars $) ;{ }^{*} p<0.05$ and $\dagger p<0.01$ different from age-matched nontransgenic controls, NewmanKeuls post hoc test. $N$, Nontransgenic; $A$, asymptomatic (at 4, 6, 8, 12 weeks of age); $O$, onset of symptoms; $E$, end-stage; $C$, cerebellum.

genic mSOD1 mice as compared with their age-matched nontransgenic controls (Fig. 1). Overall, these increases seemed most prominent for caspase-3 in early symptomatic transgenic mSOD1 mice (Fig. 1). In contrast to the spinal cord, the cerebellum from end-stage transgenic mSOD1 mice showed levels of caspase-1 and caspase-3 mRNA comparable with age-matched nontransgenic controls (data not shown). In addition, $\sim 25$-week-old transgenic wtSOD1 mice had spinal cord expression of caspase-1 and caspase-3 mRNA comparable with age-matched nontransgenic controls (data not shown).

\section{Activation of caspase-1 and caspase-3 in transgenic mSOD1 mice}

Caspase- 1 and caspase- 3 are synthesized, respectively, as 45 and 32 $\mathrm{kDa}$ full-length inactive polypeptides (Kidd, 1998). Both procaspases are cleaved during activation, generating proteolytic fragments of 20 and $10 \mathrm{kDa}$ for caspase- 1 and of 17 and $10 \mathrm{kDa}$ for caspase-3 (Kidd, 1998). In 4-week-old asymptomatic transgenic mSOD1, we detected by Western blot analysis the full-length 45 kDa caspase- 1 and $32 \mathrm{kDa}$ caspase- 3 in spinal cord extracts (Fig. 2). Over the next 8 weeks the levels of pro-caspase-1 and pro-caspase- 3 did not change significantly as compared with the nontransgenic

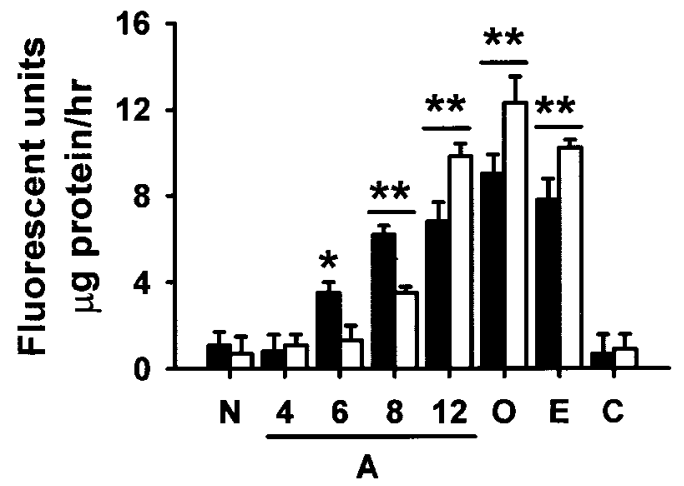

Figure 3. Increased caspase-1- and caspase-3-like activities in the spinal cords of transgenic mSOD1 mice. Caspase-1-like activity (black bars) increases before caspase-3-like activity (white bars), but both peak at the beginning of symptoms. Data are mean \pm SEM for 5-15 mice per group; ${ }^{*} p<0.05$ and ${ }^{* *} p<0.01$ higher than age-matched nontransgenic controls, Newman-Keuls post hoc test. $N$, Nontransgenic; $A$, asymptomatic (at 4, 6, 8,12 weeks of age); $O$, onset of symptoms; $E$, end-stage; $C$, cerebellum.

controls (Fig. 2). Thereafter, levels of both pro-caspases rapidly declined, reaching a nadir at the beginning of symptoms (i.e., $\sim 14$ weeks), and remained low until end-stage (i.e., $\sim 22$ weeks). In contrast, there was a significant time-related change in the levels of caspase- 1 and caspase- 3 cleavage products in the spinal cords of transgenic mSOD1 mice (Fig. 2). Neither the $20 \mathrm{kDa}$ fragment from caspase- 1 nor the $17 \mathrm{kDa}$ fragment from caspase- 3 could be detected in any of the transgenic mSOD1 mice before the age of 6 weeks (Fig. 2), yet the $20 \mathrm{kDa}$ fragment from caspase- 1 appeared by $\sim 6$ weeks of age, followed by the appearance of the $17 \mathrm{kDa}$ fragment from caspase- 3 by $\sim 10$ weeks of age (Fig. 2). From then on, both levels increased steadily over time, reaching a maximum at the beginning of symptoms and remaining significantly elevated at end-stage (Fig. 2). In the cerebellum of end-stage transgenic mSOD1 mice as well as in the spinal cords of age-matched transgenic wtSOD1 mice, levels of pro-caspase-1 and pro-caspase-3 did not differ from age-matched nontransgenic controls; no cleavage products could be detected.

\section{Increased caspase-1 and caspase-3 activity in transgenic mSOD1 mice}

In 4-week-old asymptomatic transgenic mSOD1 mice, spinal cord caspase-1-like and caspase-3-like activity was comparable with that in nontransgenic controls (Fig. 3). However, as early as 6 weeks of age, the spinal cord caspase-1-like activity in transgenic mSOD1 mice began to increase progressively until it stabilized at a maximum by the onset of symptoms (Fig. 3). By contrast, it was only by 10 weeks of age that caspase-3-like activity became elevated significantly in the spinal cords of transgenic mSOD1 mice (Fig. 3). Thereafter, caspase-3-like activity followed similar kinetics to caspase-1-like activity (Fig. 3). Both the magnitude and the rate of increase of caspase-3-like activity were greater than those of caspase-1-like activity (Fig. 3). In the cerebellum of end-stage transgenic mSOD1 mice and in the spinal cords of age-matched transgenic wtSOD1 mice, the activity of caspase- 1 and caspase- 3 did not differ from age-matched nontransgenic controls.

High expression of pro-caspase-1 and pro-caspase-3 in spinal cord motor neurons

To provide more detailed information regarding the cellular localization of caspase- 1 and caspase- 3 , we first examined the spinal cords of both transgenic mSOD1 and wild-type mice by immunohistochemistry, using antisera that recognize pro-caspase-1 or procaspase-3. In asymptomatic transgenic mSOD1 mice and their wild-type counterparts, specific immunoreactivity for both procaspases was observed in spinal cord throughout the gray matter within neurons and neuropils (Fig. $4 A, B, D, E$ ). Large motor neurons of the anterior horn showed intense pro-caspase-1 and procaspase-3 immunoreactivity (Fig. 4B,E). All other spinal cord 

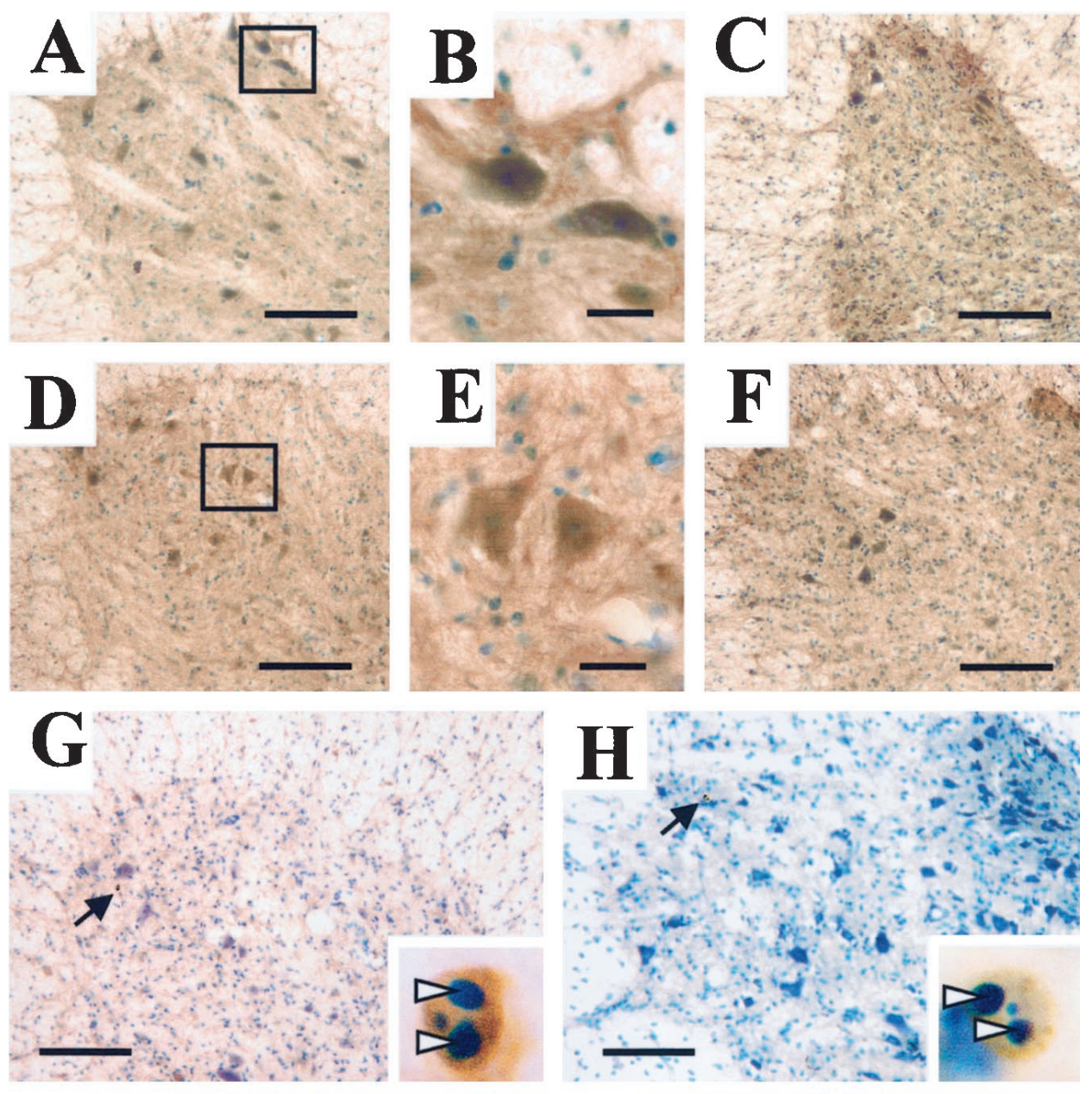

Figure 4. In nontransgenic controls a large number of cells from the lumbar segment are strongly immunoreactive for pro-caspase-1 $(A)$ and pro-caspase-3 $(D)$; those cells have a neuronal morphology (see higher magnification in $B$ and $E$ ). In end-stage transgenic mSOD1, there is a dramatic loss of procaspase-1-positive $(C)$ and pro-caspase-3positive $(F)$ neurons in the lumbar segment. Conversely, specific immunostaining for CM1 ( $G$; see arrow) and fractin ( $H$; see arrow) is seen only in symptomatic transgenic mSOD1 mice within apoptotic cells (see insets; arrowheads indicate apoptotic chromatin clumps) and colocalizes with the neuronal marker neurofilament $(I)$, but not with the glial marker GFAP $(J)$. Scale bars: $A, C, D$, $F-H, 100 \mu \mathrm{m} ; B, E, I, J, 20 \mu \mathrm{m}$.
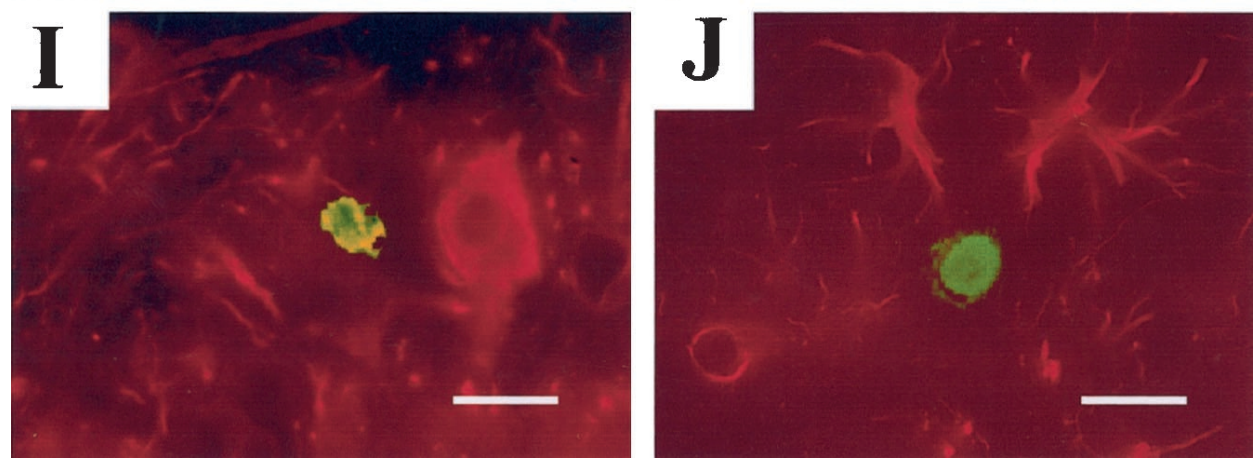

neurons showed mild pro-caspase- 1 and pro-caspase- 3 immunoreactivity (Fig. $4 A, D$ ). In early symptomatic and end-stage transgenic mSOD1 mice, the greater the loss of motor neurons, the smaller the number of pro-caspase- 1 and pro-caspase- 3 immunoreactive neurons (Fig. 4C,F).

\section{Active caspase- 3 and $\beta$-actin fragment in motor neurons of transgenic mSOD1 mice}

We also examined the spinal cords of both transgenic mSOD1 and wild-type mice by immunohistochemistry, using an antibody directed against the $17 \mathrm{kDa}$ cleavage fragment of active caspase-3 (CM1 antibody; Srinivasan et al., 1998); no antibody for active caspase-1 was available. In asymptomatic transgenic mSOD1 mice and in nontransgenic controls, CM1 immunoreactivity was barely detectable above background (Fig. $4 G$ ). By contrast, in early symptomatic and to a lesser extent in end-stage transgenic mSOD1 mice, numerous cells in the spinal cord showed strong immunoreactivity for CM1 (Table 1; Fig. 4G, inset). These CM1-positive cells were localized primarily in the anterior horn and had a morphology reminiscent of neurons. Confirming their neuronal origin is the fact that most CM1-positive cells were immunoreactive for neurofilament, whereas none were immunoreactive for the astrocytic marker glial fibrillary acidic protein (GFAP) or the microglial marker macrophage antigen-1 (MAC-1; Fig. 4G,I). Furthermore, all CM1-positive neurons exhibited definite morphology of apoptotic neurons (Macaya et al., 1994) in that they showed small cell bodies and nuclei with variable numbers of basophilic nucleic chromatin clumps (Fig. 4G, inset). No CM1-positive cells could be detected in the cerebellum of end-stage transgenic mSOD1 mice or the spinal cord of age-matched transgenic wtSOD1 mice.

To confirm that CM1 immunostaining reflected active caspase-3, we immunostained spinal cord sections with an antibody directed against fractin, a protein fragment that is generated specifically after the cleavage of $\beta$-actin by active caspase-3 (Yang et al., 1998). In asymptomatic transgenic mSOD1 mice and nontransgenic controls, no fractin immunoreactive cells were observed. In contrast, in both early symptomatic and end-stage transgenic mSOD1 mice, 
Table 1. Spinal cord cells containing activated caspase-3 and $\beta$-actin fragment

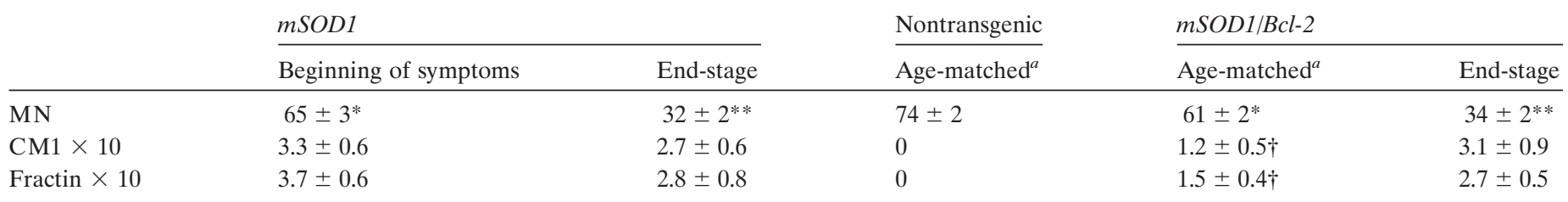

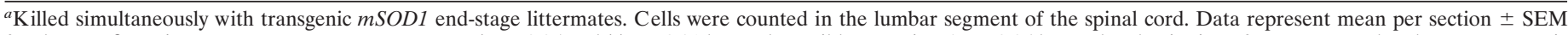

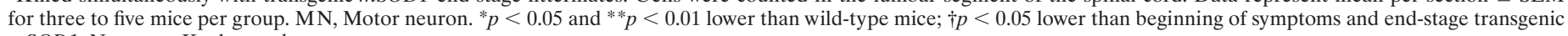
mSOD1, Newman-Keuls post hoc test.

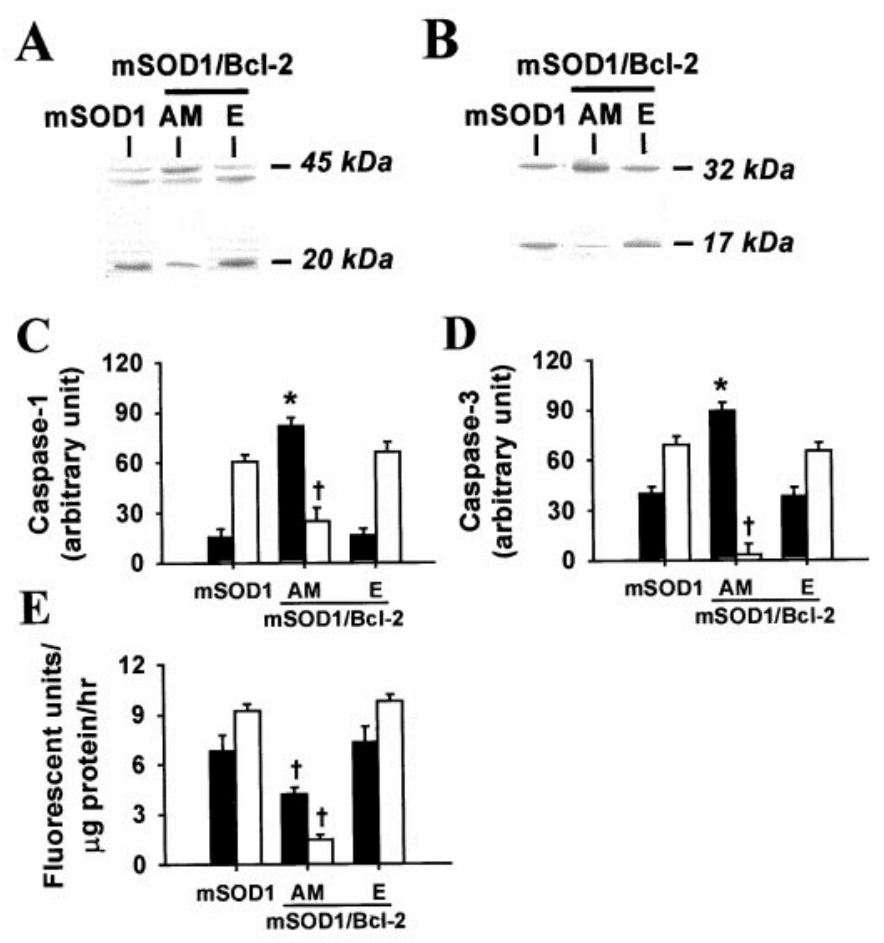

Figure 5. Bcl-2 delays caspase activation. Western blot analyses $(A-D)$ show that transgenic mSOD1/Bcl-2 mice that are aged-matched $(A M)$ with end-stage $(E)$ transgenic mSOD1 exhibit significantly lower levels of cleaved caspase-1 $(A, C)$ and caspase-3 $(B, D)$ than end-stage transgenic mSOD1. However, end-stage transgenic mSOD1/Bcl-2 mice exhibit levels of cleaved caspase-1 $(A, C)$ and caspase-3 $(B, D)$ comparable with end-stage transgenic mSOD1. A similar situation is found for the activity of caspase-1 and caspase-3 $(E)$; ${ }^{*} p<0.05$ higher and $\dagger p<0.05$ lower than end-stage transgenic mSOD1 and mSOD1/Bcl-2 mice, Newman-Keuls post hoc test.

numerous fractin immunoreactive cells exhibiting similar morphological characteristics to CM1-positive cells were found in the anterior horn (Table 1; Fig. 4H, inset).

\section{$\mathrm{Bcl}-2$ overexpression delays caspase activation in transgenic mSOD1 mice}

Although transgenic mSOD1 mice reached end-stage by $\sim 25$ weeks of age, at that time transgenic mSOD1/Bcl-2 mice were affected only minimally and reached end-stage much later, by 30 weeks of age. Contrasting with end-stage transgenic mSOD1 mice, agematched transgenic mSOD1/Bcl-2 mice showed minimal cleavage of caspase-1 or caspase-3 and diminished caspase-1- and caspase3-like activity (Fig. 5). In addition, there were significantly fewer CM1- and fractin-positive cells in 25-week-old transgenic mSOD1/ Bcl-2 mice than in end-stage transgenic mSOD1 mice (Table 1). However, end-stage 30-week-old transgenic mSOD1/Bcl-2 mice had levels of caspase cleavage products, caspase-like activity, and numbers of CM1- and fractin-positive cells comparable with 25week-old end-stage transgenic mSOD1 (Table 1; Fig. 5).

\section{DISCUSSION}

The present study shows that both pro-caspase- 1 and -3 are expressed constitutively in the spinal cords of normal mice (see Fig. 4 ) and that caspase- 1 and -3 mRNA levels are unchanged or slightly increased during the course of the disease in transgenic mSOD1 mice (see Fig. 1). Immunolabeling for both pro-caspases was found in the neuropil and within numerous cells with a neuronal morphology throughout the gray matter of the spinal cords. Procaspase immunoreactivity was most intense in large motor neurons of the anterior horn, the known primary targets of the neurodegenerative process in ALS. In contrast to the abundance of procaspase-positive neurons in the anterior horn of normal animals, only a few of these neurons were seen in early symptomatic transgenic mSOD1 mice and even fewer in end-stage transgenic mSOD1 mice (see Fig. 4). This observation is consistent with the fact that spinal cord of symptomatic transgenic mSOD1 mice is the site of a dramatic loss of primarily, but not exclusively, large motor neurons (Morrison et al., 1996).

This study also shows pro-caspase- 1 and pro-caspase- 3 cleavage products and increased caspase-1-like and caspase-3-like activity in spinal cord homogenates of transgenic mSOD1 mice during the neurodegenerative process (see Figs. 2, 3). Active caspase-1 and caspase- 3 were found only in diseased areas of the nervous system of transgenic mSOD1 mice, because cerebellum, which is devoid of neuropathological changes in this model (Dal Canto and Gurney, 1995), did not show any evidence of pro-caspase cleavage products or increased caspase-like activity in end-stage transgenic mSOD1 mice (see Fig. 2). We also demonstrate that caspase activation is related to the expression of the mutant protein and not to increased SOD1 enzymatic activity, because age-matched transgenic wtSOD1 mice showed no pro-caspase cleavage products and no increased caspase-like activity in any region that was studied (data not shown). Activation of both caspases culminated in 14-week-old transgenic mSOD1 mice (see Figs. 2, 3), the age at which the most active wave of motor neuron death occurs in these animals (Kong and $\mathrm{Xu}, 1998)$. Given the essential role played by active caspases, and especially active caspase- 3 , in neuronal death in a large variety of experimental and pathological situations, our data suggest that caspase- 1 and caspase- 3 activation could be instrumental in mSOD1-induced neurodegeneration.

Of note, active caspase- 1 was detected in the spinal cord of transgenic mSOD1 mice $\sim 4$ weeks before any evidence of caspase- 3 activation by immunoblot and enzymatic assay (see Figs. $2,3)$. Activation of caspase- 1 preceding that of caspase- 3 has been observed previously in a mouse lymphoma-derived cell line subjected to Fas activation (Enari et al., 1996) and in rat hippocampal neuronal cultures exposed to staurosporine (Krohn et al., 1998). The appearance of active caspase- 1 before active caspase- 3 could indicate that, in this mouse model of ALS, caspase activation proceeds in a sequential manner. It also suggests the possibility that caspase- 3 activation depends on the previous presence of caspase1-like activity. Relevant to this is the demonstration that active caspase- 1 can cleave pro-caspase-3, thus activating caspase-3 (Xue et al., 1996).

Early activation of caspase-1 occurred while transgenic mSOD1 mice still showed no gross behavioral abnormalities or neuronal loss (Kong and $\mathrm{Xu}, 1998$ ). This observation raises the question as 
to the role of active caspase- 1 in the neurodegenerative process in transgenic mSOD1 mice. Aside from its role in cell death, active caspase- 1 and the product of its substrate, the cytokine interleukin$1 \beta$, possess pro-inflammatory properties ( $\mathrm{Li}$ et al., 1995). However, it is unlikely that the early appearance of active caspase- 1 is involved primarily in inflammation because inflammatory events such as gliosis arise much later in the course of the disease, paralleling but not preceding the loss of motor neurons (Almer et al., 1999; Levine et al., 1999). Alternatively, in the chronic neurodegenerative disorder Huntington's disease, the inhibition of caspase- 1 delays the appearance of neuronal inclusions, neurotransmitter receptor alterations, and the onset of symptoms (Ona et al., 1999), indicating a role for caspase-1 in neuronal dysf unction in this condition. A similar scenario can apply to active caspase- 1 in transgenic mSOD1 mice because, before any detectable loss of motor neurons, these animals exhibit a marked decrement in their motor abilities (Kong and Xu, 1998).

During the neurodegenerative process in transgenic mSOD1 mice, aside from the loss of neurons, there is also an intense glial reaction (Almer et al., 1999). It is thus important to emphasize that active caspase-3, as evidenced by CM1 immunostaining, was found essentially within neurons and not within glial cells in the anterior horn of the spinal cords of symptomatic transgenic mSOD1 mice (see Fig. 4). The number of active caspase-3-positive neurons was greater in early symptomatic than in end-stage transgenic mSOD1 mice (Table 1), a finding that agrees with the magnitude of caspase cleavage and enzymatic activity found at these two stages of the disease (see Figs. 2, 3). However, although the absolute number of active caspase-3-positive neurons decreased during the progression of the neurodegenerative process (see Table 1), their proportion relative to the number of remaining motor neurons increased, suggesting that more neurons are actually dying at end-stage as compared with the beginning of symptoms.

Active caspase-3 was found within neurons showing shrunken cell bodies and nuclei and prominent basophilic chromatin clumps (see Fig. 4), all hallmarks of apoptosis (Jackson-Lewis et al., 2000). Relevant to this finding is the demonstration of caspase- 3 activation within apoptotic motor neurons in the anterior horn of the spinal cord and in the motor cortex from human ALS postmortem samples (Martin, 1999). However, although unquestionably present, apoptotic cells in these animals were not abundant (see Table 1) and thus easily can be missed (Migheli et al., 1999). Because the half-life of apoptotic cells is short (Wyllie et al., 1980), one cannot extrapolate from their numbers either the number of neurons dying by apoptosis or the number of motor neurons dying at any given time. Furthermore, a previous study (Chiu et al., 1995) reported that only a small number of motor neurons die at any given time in the spinal cord of affected transgenic mSOD1 mice. Consequently, we would not expect to find a much greater number of apoptotic profiles per tissue section than what is reported in Table 1. Accordingly, the scarcity of apoptotic cells in transgenic mSOD1 mice cannot undermine the importance of apoptosis in the death of motor neurons in this model of ALS. In addition, it should be made clear that our data do not exclude the contribution of a nonapoptotic mode of cell death such as necrosis in the overall demise of spinal cord motor neurons in affected transgenic mSOD1 mice, especially at end-stage.

It is also worth emphasizing that the small number of apoptotic neurons contrasts with the large increases in levels of activated caspases. In these animals, there are prominent morphological alterations in mitochondria (Kong and Xu, 1998), suggesting that most of the activation of the caspases might be occurring in neuronal processes and synapses (Mattson and Duan, 1999). Therefore, it is possible that the discrepancy between our immunohistochemical and enzymatic assay findings stems from the fact that the former method only looked at cell bodies whereas the latter looked at whole tissue, including neurons and neuropils.

During the cell death execution active caspase-3 cleaves several intracellular proteins (Thornberry and Lazebnik, 1998; Yang et al., 1998). Although the relevance of certain of these cleaved molecules in the dying process is uncertain, their demonstration can serve as a cellular footprint of caspase-3 activation. In this vein, we have studied fractin, a product of caspase-3-related cleavage of $\beta$-actin, which correlates with the occurrence of apoptosis and of caspase-3 activation in different cell death settings (Yang et al., 1998; Suurmeijer et al., 1999). In both early symptomatic and end-stage transgenic mSOD1 mice, we found numerous fractinimmunostained cells that were localized to the anterior horn of the spinal cord and that exhibited the exact same morphological characteristics as described above for active caspase-3-positive cells (see Fig. 4). This finding provides meaningful functional information, which allows one to conclude that caspase- 3 activation may have real pathological consequences in this model of ALS.

Compared with end-stage transgenic mSOD1 mice, age-matched transgenic $\mathrm{mSOD} 1 / \mathrm{Bcl}-2$ mice not only were minimally symptomatic but had almost no caspase activation in the spinal cord (see Figs. 2, 3). Thus, overexpression of Bcl-2 prevents the activation of both caspase- 1 and -3 in the spinal cord of transgenic mSOD1 mice, which is consistent with the known central role of Bcl-2 in regulating caspase activation (Pettmann and Henderson, 1998). Nevertheless, even in the presence of increased levels of Bcl-2, transgenic mSOD1/Bcl-2 mice eventually became paralyzed and had marked caspase activation in the spinal cord (see Figs. 2, 3). Therefore, overexpression of Bcl-2, as previously noted (Kostic et al., 1997), does not block but rather delays mSOD1-mediated deleterious effects. The transient nature of the Bcl-2 beneficial actions may be attributable to the fact that, during the neurodegenerative process, the fine-tuned balance between repressors and promoters of neuronal death is progressively upset, favoring the pro-neuronal death forces. Relevant to this is our demonstration that Bax, a Bcl-2 family member that promotes neuronal death (Merry and Korsmeyer, 1997), is upregulated gradually in transgenic mSOD1 mice during the disease progression (Vukosavic et al., 1999).

Collectively, our study provides compelling evidence for caspase activation in the transgenic mSOD1 mouse model of ALS. Given these findings, it may be proposed that the inhibition of caspase is a valuable target for the development of therapies for ALS aimed at slowing the progression of the neurodegenerative process. Major impetus for this view is found in our recent demonstration that the chronic infusion of a pan-caspase inhibitor provides marked beneficial effects in transgenic mSOD1 mice (Li et al., 2000). However, this study also shows that the inhibition of caspases may not be sufficient to stop the disease. Accordingly, the ideal therapeutic approach for ALS may rely on the combination of anti-apoptotic compounds with other agents that have beneficial effects in this model of ALS, such as vitamin E, riluzole, and creatine (Gurney et al., 1996; Klivenyi et al., 1999).

\section{REFERENCES}

Almer G, Vukosavic S, Romero N, Przedborski S (1999) Inducible nitric oxide synthase upregulation in a transgenic mouse model of familial amyotrophic lateral sclerosis. J Neurochem 72:2415-2425.

Ara J, Przedborski S, Naini AB, Jackson-Lewis V, Trifiletti RR, Horwitz J, Ischiropoulos H (1998) Inactivation of tyrosine hydroxylase by nitration following exposure to peroxynitrite and 1-methyl-4-phenyl-1,2,3,6tetrahydropyridine (MPTP). Proc Natl Acad Sci USA 95:7659-7663.

Brown Jr RH (1995) Superoxide dismutase in familial amyotrophic lateral sclerosis: models for gain of function. Curr Opin Neurobiol 5:841-846.

Chiu AY, Zhai P, Dal Canto MC, Peters T, Kwon YH, Prattis SM, Gurney ME (1995) Age-dependent penetrance of disease in a transgenic mouse model of familial amyotrophic lateral sclerosis. Mol Cell Neurosci $6: 349-362$

Coggeshall RE, Lekan HA (1996) Methods for determining numbers of cells and synapses: a case for more uniform standards of review. J Comp Neurol 364:6-15.

Dal Canto MC, Gurney ME (1995) Neuropathological changes in two lines of mice carrying a transgene for mutant human $\mathrm{Cu} / \mathrm{Zn} \mathrm{SOD}$, and in mice overexpressing wild-type human SOD: a model of familial amyotrophic lateral sclerosis (FALS). Brain Res 676:25-40.

Deng H-X, Hentati A, Tainer JA, Iqbal Z, Cayabyab A, Hung W-Y, Getzoff ED, Hu P, Herzfeldt B, Roos RP, Warner C, Deng G, Soriano E, Smyth C, Parge HE, Ahmed A, Roses AD, Hallewell RA, PericakVance MA, Siddique T (1993) Amyotrophic lateral sclerosis and structural defects in $\mathrm{Cu} / \mathrm{Zn}$ superoxide dismutase. Science 261:1047-1051.

Durham HD, Roy J, Dong L, Figlewicz DA (1997) Aggregation of mutant 
$\mathrm{Cu} / \mathrm{Zn}$ superoxide dismutase proteins in a culture model of ALS. J Neuropathol Exp Neurol 56:523-530.

Enari M, Talanian RV, Wong WW, Nagata S (1996) Sequential activation of ICE-like and CPP32-like proteases during Fas-mediated apoptosis. Nature 380:723-726.

Estevez AG, Crow JP, Sampson JB, Reiter C, Zhuang Y, Richardson GJ Tarpey MM, Barbeito L, Beckman JS (1999) Induction of nitric oxidedependent apoptosis in motor neurons by zinc-deficient superoxide dismutase. Science 286:2498-2500.

Friedlander RM, Brown RH, Gagliardini V, Wang J, Yuan J (1997) Inhibition of ICE slows ALS in mice. Nature 388:31.

Gillardon F, Bottiger B, Schmitz B, Zimmermann M, Hossmann KA (1997) Activation of CPP-32 protease in hippocampal neurons following ischemia and epilepsy. Brain Res Mol Brain Res 50:16-22.

Gurney ME, Pu H, Chiu AY, Dal Canto MC, Polchow CY, Alexander DD, Caliendo J, Hentati A, Kwon YW, Deng H-X, Chen W, Z hai P, Sufit RL, Siddique T (1994) Motor neuron degeneration in mice that express a human $\mathrm{Cu} / \mathrm{Zn}$ superoxide dismutase mutation. Science 264:1772-1775.

Gurney ME, Cutting FB, Zhai P, Doble A, Taylor CP, Andrus PK, Hall ED (1996) Benefit of vitamin E, riluzole, and gabapentin in a transgenic model of familial amyotrophic lateral sclerosis. Ann Neurol 39:147-157.

Hakem R, Hakem A, Duncan GS, Henderson JT, Woo M, Soengas MS, Elia A, De la Pompa JL, Kagi D, Khoo W, Potter J, Yoshida R, Kaufman SA, Lowe SW, Penninger JM, Mak TW (1998) Differential requirement for caspase-9 in apoptotic pathways in vivo. Cell 94:339-352.

Jackson-Lewis V, Vila M, Djaldetti R, Guegan C, Liberatore G, Liu J O’Malley KL, Burke RE, Przedborski S (2000) Developmental cell death in dopaminergic neurons of the substantia nigra of mice. J Comp Neurol 424:476-488.

Kidd VJ (1998) Proteolytic activities that mediate apoptosis. Annu Rev Physiol 60:533-573.

Klivenyi P, Ferrante RJ, Matthews RT, Bogdanov MB, Klein AM, Andreassen OA, Mueller G, Werner M, Kaddurah-Daouk R, Beal MF (1999) Neuroprotective effects of creatine in a transgenic animal model of amyotrophic lateral sclerosis. Nat Med 5:347-350.

Kong JM, Xu ZS (1998) Massive mitochondrial degeneration in motor neurons triggers the onset of amyotrophic lateral sclerosis in mice expressing a mutant SOD1. J Neurosci 18:3241-3250.

Kostic V, Jackson-Lewis V, De Bilbao F, Dubois-Dauphin M, Przedborski $\mathrm{S}$ (1997) Bcl-2: prolonging life in a transgenic mouse model of familial amyotrophic lateral sclerosis. Science 277:559-562.

Krohn AJ, Preis E, Prehn JH (1998) Staurosporine-induced apoptosis of cultured rat hippocampal neurons involves caspase-1-like proteases as upstream initiators and increased production of superoxide as a main downstream effector. J Neurosci 18:8186-8197.

Kuida K, Zheng TS, Na S, Kuan C, Yang D, Karasuyama H, Rakic P, Flavell RA (1996) Decreased apoptosis in the brain and premature lethality in CPP32-deficient mice. Nature 384:368-372.

Kuida K, Haydar TF, Kuan CY, Gu Y, Taya C, Karasuyama H, Su MS, Rakic P, Flavell RA (1998) Reduced apoptosis and cytochrome $c$-mediated caspase activation in mice lacking caspase-9. Cell 94:325-337.

Kunst CB, Mezey E, Brownstein MJ, Patterson D (1997) Mutations in SOD1 associated with amyotrophic lateral sclerosis cause novel protein interactions. Nat Genet 15:91-94.

Levine JB, Kong J, Nadler M, Xu Z (1999) Astrocytes interact intimately with degenerating motor neurons in mouse amyotrophic lateral sclerosis (ALS). Glia 28:215-224.

Li M, Ona VO, Guegan C, Chen M, Jackson-Lewis V, Andrews LJ, Olszewski AJ, Stieg PE, Lee JP, Przedborski S, Friedlander RM (2000) Functional role of caspase-1 and caspase- 3 in an ALS transgenic mouse model. Science 288:335-339.

Li P, Allen H, Banerjee S, Franklin S, Herzog L, Johnston C, McDowell J, Paskind M, Rodman L, Salfeld J, Towne E, Tracey D, Wardwell S, Wei F-Y, Wong W (1995) Mice deficient in IL-1 $\beta$-converting enzyme are defective in production of mature IL-1 $\beta$ and resistant to endotoxic shock. Cell 80:401-411.

Macaya A, Munell F, Gubits RM, Burke RE (1994) Apoptosis in substantia nigra following developmental striatal excitotoxic injury. Proc Nat Acad Sci USA 91:8117-8121.

Mandir AS, Przedborski S, Jackson-Lewis V, Wang ZQ, SimbulanRosenthal M, Smulson ME, Hoffman BE, Guastella DB, Dawson VL, Dawson TM (1999) Poly (ADP-ribose) polymerase activation mediates MPTP-induced parkinsonism. Proc Natl Acad Sci USA 96:5774-5779.

Martin LJ (1999) Neuronal death in amyotrophic lateral sclerosis is apoptosis: possible contribution of a programmed cell death mechanism. J Neuropathol Exp Neurol 58:459-471.

Martinou J-C, Dubois-Dauphin M, Staple JK, Rodriguez I, Frankowski H, Missotten M, Albertinini P, Talabot D, Catsicas S, Pietra C, Huarte J (1994) Overexpression of bcl-2 in transgenic mice protects neurons from naturally occurring cell death and experimental ischemia. Neuron 13:1017-1030.

Mattson MP, Duan W (1999) “Apoptotic" biochemical cascades in synaptic compartments: roles in adaptive plasticity and neurodegenerative disorders. J Neurosci Res 58:152-166.
Mena MA, Khan U, Togasaki DM, Sulzer D, Epstein CJ, Przedborski S (1997) Effects of wild-type and mutated copper/zinc superoxide dismutase on neuronal survival and L-DOPA-induced toxicity in postnatal midbrain culture. J Neurochem 69:21-33.

Merry DE, Korsmeyer SJ (1997) Bcl-2 gene family in the nervous system. Annu Rev Neurosci 20:245-267.

Migheli A, Atzori C, Piva R, Tortarolo M, Girelli M, Schiffer D, Bendotti C (1999) Lack of apoptosis in mice with ALS. Nat Med 5:966-967.

Morrison BM, Gordon JW, Ripps ME, Morrison JH (1996) Quantitative immunocytochemical analysis of the spinal cord in G86R superoxide dismutase transgenic mice: neurochemical correlates of selective vulnerability. J Comp Neurol 373:619-631.

Namura S, Zhu J, Fink K, Endres M, Srinivasan A, Tomaselli KJ, Yuan J, Moskowitz MA (1998) Activation and cleavage of caspase-3 in apoptosis induced by experimental cerebral ischemia. J Neurosci 18:3659-3668.

Ona VO, Li M, Vonsattel JP, Andrews LJ, Khan SQ, Chung W M, Frey AS, Menon AS, Li XJ, Stieg PE, Yuan J, Penney JB, Young AB, Cha JH, Friedlander RM (1999) Inhibition of caspase-1 slows disease progression in a mouse model of Huntington's disease [see comments]. Nature 399:263-267.

Pasinelli P, Borchelt DR, Houseweart MK, Cleveland DW, Brown RHJ (1998) Caspase-1 is activated in neural cells and tissue with amyotrophic lateral sclerosis-associated mutations in copper/zinc superoxide dismutase. Proc Natl Acad Sci USA 95:15763-15768.

Pettmann B, Henderson CE (1998) Neuronal cell death. Neuron 20:633-647.

Przedborski S, Jackson-Lewis V, Yokoyama R, Shibata T, Dawson VL, Dawson TM (1996) Role of neuronal nitric oxide in MPTP (1-methyl4-phenyl-1,2,3,6-tetrahydropyridine)-induced dopaminergic neurotoxicity. Proc Natl Acad Sci USA 93:4565-4571.

Rabizadeh S, Gralla EB, Borchelt DR, Gwinn R, Valentine JS, Sisodia S, Wong P, Lee M, Hahn H, Bredesen DE (1995) Mutations associated with amyotrophic lateral sclerosis convert superoxide dismutase from an anti-apoptotic gene to a proapoptotic gene: studies in yeast and neural cells. Proc Natl Acad Sci USA 92:3024-3028.

Rosen DR, Siddique T, Patterson D, Figlewicz DA, Sapp P, Hentati A, Donaldson D, Goto J, O'Regan JP, Deng H-X, Rahmani Z, Krizus A, McKenna-Yasek D, Cayabyab A, Gaston SM, Berger R, Tanzi RE, Halperin JJ, Herzfeldt B, Van den Bergh R, Hung W-Y, Bird T, Deng G, Mulder DW (1993) Mutations in $\mathrm{Cu} / \mathrm{Zn}$ superoxide dismutase gene are associated with familial amyotrophic lateral sclerosis. Nature 362:59-62.

Rowland LP (1995) Hereditary and acquired motor neuron diseases. In: Merritt's textbook of neurology (Rowland LP, ed), pp 742-749. Philadelphia: Williams \& Wilkins.

Srinivasan A, Roth KA, Sayers RO, Shindler KS, Wong AM, Fritz LC, Tomaselli KJ (1998) In situ immunodetection of activated caspase-3 in apoptotic neurons in the developing nervous system. Cell Death Differ 5:1004-1016.

Stefanis L, Park DS, Yan CYI, Farinell SE, Troy CM, Shelanski ML, Greene LA (1996) Induction of CPP32-like activity in PC12 cells by withdrawal of trophic support: dissociation from apoptosis. J Biol Chem 271:30663-30671.

Suurmeijer AJ, van der Wijk J, van Veldhuisen DJ, Yang F, Cole GM (1999) Fractin immunostaining for the detection of apoptotic cells and apoptotic bodies in formalin-fixed and paraffin-embedded tissue. Lab Invest 79:619-620.

Thornberry NA, Lazebnik YA (1998) Caspases: enemies within. Science 281:1312-1316.

Vukosavic S, Dubois-Dauphin M, Romero N, Przedborski S (1999) Bax and Bcl-2 interaction in a transgenic mouse model of familial amyotrophic lateral sclerosis. J Neurochem 73:2460-2468.

Wiedau-Pazos M, Goto JJ, Rabizadeh S, Gralla EB, Roe JA, Lee MK, Valentine JS, Bredesen DE (1996) Altered reactivity of superoxide dismutase in familial amyotrophic lateral sclerosis. Science 271:515-518.

Wyllie AH, Kerr JF, Currie AR (1980) Cell death: the significance of apoptosis. Int Rev Cytol 68:251-306.

Xue D, Shaham S, Horvitz HR (1996) The Caenorhabditis elegans celldeath protein CED-3 is a cysteine protease with substrate specificities similar to those of the human CPP32 protease. Genes Dev 10:1073-1083.

Yakovlev AG, Knoblach SM, Fan L, Fox GB, Goodnight R, Faden AI (1997) Activation of CPP32-like caspases contributes to neuronal apoptosis and neurological dysfunction after traumatic brain injury. J Neurosci 17:7415-7424.

Yang F, Sun X, Beech W, Teter B, Wu S, Sigel J, Vinters HV, Frautschy SA, Cole GM (1998) Antibody to caspase-cleaved actin detects apoptosis in differentiated neuroblastoma and plaque-associated neurons and microglia in Alzheimer's disease. Am J Pathol 152:379-389.

Yim MB, Kang JH, Yim HS, Kwak HS, Chock PB, Stadtman ER (1996) A gain-of-function of an amyotrophic lateral sclerosis-associated $\mathrm{Cu} / \mathrm{Zn}$ superoxide dismutase mutant: an enhancement of free radical formation due to a decrease in $K_{\mathrm{m}}$ for hydrogen peroxide. Proc Natl Acad Sci USA 93:5709-5714. 